\title{
What happens when organisations embrace social networking? Knowledge sharing at a multinational business solutions corporation
}

\author{
C. Stafford \\ Centre for Information and Knowledge Management, University of Johannesburg \\ South Africa \\ catherine_s@live.co.za
}

\section{M.A. Mearns}

Department of Information and Knowledge Management, University of Johannesburg

South Africa

mearnsm@uj.ac.za

Amid widespread resistance to online social networking tools, their effectiveness in promoting knowledge sharing in a knowledgedriven organisation was demonstrated in the study. Usage patterns, user attitudes and perceptions regarding online social networking technologies as a professional application for knowledge sharing within the workplace were investigated. Selfadministered questionnaires were administered to a sample of IBM Global Business Services employees in South Africa. Upon completion of the questionnaire analysis an interview was conducted with the knowledge manager for verification and clarification purposes. The results revealed the respondents' positive attitudes regarding the use of social networking tools for knowledge sharing. The culture of knowledge sharing at IBM and the contribution that social networking tools makes within the company were uncovered. Findings disclosed that the online social networking tools were effective and that management at IBM encourages employees to make more and more use of the tools for knowledge sharing and knowledge creation. The results of this study demonstrate the effectiveness of online social networking tools and serve as encouragement to hesitant organisations to adopt social networking in their business practices.

Keywords: Online social networking; knowledge sharing; IBM, competitive advantage; innovation

Received: 7 Dec. 2009

\section{Contents}

1 Introduction

2 Knowledge sharing in the knowledge economy

3 Methodology design

4 Online social networking at IBM

4.1 Awareness, use and preference of online social networking at IBM

4.2 Applications of online social networking tools at IBM

4.3 Knowledge sharing as an organisational resource

4.4 Social networking and its importance for organisations

4.5 Adoption of social networking in the workplace

5 Conclusion

6 Acknowledgement

7 References 


\section{Introduction}

The only thing that gives an enterprise an advantage over others is what it knows, how it uses what it knows and how quickly it can know something new. (Prusak 1996:6)

Knowledge sharing is a concept that has always intrigued practitioners and researchers in the knowledge management discipline and significant research has been conducted into understanding what hampers knowledge sharing and what makes it successful (Chua 2003:117). In current times of continual change, the success of any organisation lies in its ability to utilise knowledge-sharing practices and procedures to create, disseminate and use new knowledge in order to outlive or outlast the competition (Van der Merwe 2004, Van der Walt 2003). Organisations that are able to access their employees' knowledge are those that understand what knowledge is, where it lies and how it can be exploited (Van der Walt 2003). In many organisations, knowledge sharing takes place through informal networks or organisational communication networks and it is through these informal networks, rather than through formal graphs and diagrams, that value-adding information is created (Liebowitz 2007). Knowledge sharing can be beneficial to organisations operating in the knowledge economy. The focus of organisational resources has shifted from tangible resources in the form of books and journals to intangible knowledge sources with the emphasis on tacit knowledge. The ability of an organisation to capture and use these intangible resources adds value to the company and increases the competitive advantage in an economy driven by knowledge. The ability of an organisation to understand where its information and knowledge is coming from and how it is most effectively created lies in the organisation's capacity to create an environment where knowledge sharing and collaboration are promoted.

The use of online social networks (OSNs) has changed the way in which people communicate on a social and professional level. The impact that OSN technologies have had on the way that people interact is not only evident in the growing number of tools that one can subscribe to, but also in the unprecedented growth in the number of subscribers to such technologies. OSN technologies offer a powerful environment in which knowledge sharing and collaboration can take place - one that transcends traditional internal organisational and divisional boundaries as well as physical boundaries in organisations situated in geographically dispersed locations. The use of OSN technologies in organisations, on a professional rather than a social level, is becoming increasingly critical as a way of tapping into the minds of employees and accessing new and innovative ideas and knowledge. Positive attitudes towards and perceptions of the use of OSN tools within organisations can contribute to the conservation and protection of knowledge, and to the enhancement of knowledge sharing and learning among employees. It is argued that knowledge sharing within organisations can be greatly improved through the use of OSN technologies and have a positive spin-off in terms of employee productivity through the creation of online communities of practice (Ferreira and Du Plessis 2009).

However, many organisations remain sceptical about the use of OSN technologies within the company because of the perception that employees will abuse the tools for social purposes rather than for organisational knowledge sharing (McClure 2009:2). There is also a perception that the use of OSN technologies is a waste of time and resources and that private company information could fall into the wrong hands, threatening the competitive advantage of the organisation. However, it is through the use of tools such as blogs, Wikis, forums, virtual communities and instant messaging that employees are able to collaborate and share their ideas and knowledge in an informal setting.

This article argues that OSN tools are highly effective in promoting knowledge sharing on a professional level among employees, especially in organisations that are knowledge dependent within an economy that is driven by knowledge.

One such organisation that has adopted the use of OSN tools throughout the entire organisation is IBM. The empirical research reported in this article was conducted at the Global Business Services (GBS) sector of IBM South Africa. The research findings presented in this article address the perceived scepticism about OSN tools in the workplace and demonstrate both the benefits and the disadvantages of the use of such tools for knowledge sharing. 


\section{Knowledge sharing in the knowledge economy}

In the knowledge economy, the creation and use of knowledge replaces labour, raw material and capital as the primary resource of production, and the emphasis is on the ability to gather, create and use knowledge effectively (Clarke 2001:189, Van der Merwe 2004). A knowledge-based economy is one in which 'firms search for linkages to promote inter-firm interactive learning and for outside partners and networks to provide complementary assets' (Clarke 2001:189). Where the focus in the industrial economy has always been on manual labour and the workforce, a different set of skills and expertise is needed in the knowledge economy. Information and communication technologies have driven change in the knowledge economy and have changed the face of business. As a result, the workforce of the knowledge economy needs to be highly skilled and advanced in their thinking and technical abilities (Brooking 1996:1). There are three primary drivers of a knowledge economy, namely, globalisation, information technology and shareholder value (Van der Merwe 2004). OSN technologies support at least the first two of these drivers. OSN is an information technology that transcends any international boundary through the use of Web 2.0 applications such as instant messaging, Wikis, blogs, video and podcasts, real simple syndication (RSS) and file sharing.

Information professionals were some of the first to embrace Web 2.0 technologies (Warr 2008:599). Since Web 2.0 was coined in 2004 much research has been conducted on the effect, advantages, disadvantages and challenges facing users, administrators and organisations to manage the growing popularity of OSN technologies (Ferreira and Du Plessis 2009, Goetchius 2008, Liebowitz 2007, McClure 2009, Reid 2009). The ability of people to collaborate and interact with information, self-publish, share expertise and find resources using Web 2.0 technologies is deemed an easier and cheaper system than any other earlier knowledge management system (Warr 2008:602). Research points to the need for Web 2.0 technologies to be managed very carefully for it to reach its full potential as a collaboration business tool (Schneckenberg 2009:234). Some of the recommendations of such research are that employees should be empowered to use Web 2.0 technologies as knowledge management systems. Furthermore, governance structures of organisations should create liberal work cultures to successfully implement Web 2.0 for knowledge sharing (Schneckenberg 2009:234).

Organisations in the knowledge economy focus on the knowledge they possess as well as on how they can capture and use that knowledge to the benefit of their organisation. Knowledge has become a resource without which no business can be successful, and the sharing of that knowledge has become a vital and natural part of business for many organisations. Organisations have had to learn how to create environments where employees are willing to share their ideas, thoughts, experiences and expertise for others to learn from.

The primary aim for organisations in the knowledge economy should be to exploit knowledge-sharing practices in order to build their intellectual capital base (Zhou and Fink 2003:39). In many cases, knowledge sharing within organisations takes place informally between employees and through the organisation's communication networks. It is because of this that the notion of using social networking within organisations for knowledge sharing was developed. The idea was that if employees would use these networks to share their knowledge and collaborate on an informal level then these tools should be available as part of a systematic process within the company (Van der Walt 2003). Organisations that are successful in accessing the knowledge of its employees are those that understand what knowledge is and know how they can exploit it (Van der Walt 2003), thus leading to innovation.

Innovation is about the purposeful application of information, creativity and initiative in creating a different value from resources that surpasses that created by their competitors (Business Dictionary 2009). In the knowledge economy, the ability of organisations to be innovative and creative and thus to create new and better products than those of their competitors enables them to gain a competitive advantage. According to Amidon (1999), innovation, more specifically knowledge innovation, is 'the creation, evolution, exchange and application of new ideas into marketable goods and services'. Knowledge innovation is more applicable in the knowledge economy as it encompasses the use of the knowledge of employees to develop new and creative products.

In a company, informal networks are conducive to knowledge sharing. Such networks are generally established casually when people meet regularly, for instance at lunchtime, when they fetch coffee or water, or when they visit across departments and 
beyond divisional boundaries. The connections that are made through informal human networks using OSN have the ability to increase the organisational intelligence of individuals (Liebowitz 2007:1). These connections remain undocumented and although tacit knowledge is exchanged there is still no tangible evidence of such interactions.

The Internet has changed the way people communicate. One of the core means of communication in the knowledge economy is through the use of the Internet and, more specifically, 'within the confines of a social network' (Goetchius 2008:5). Large groups of people can communicate effortlessly and efficiently through social networks, and the online, virtual experience is unlike anything the world has ever experienced. The creation of new knowledge is one of the main reasons for companies showing an interest in social networking (Liebowitz 2007:11). Admittedly, as many authors have pointed out, social networking has risks and disadvantages, such as causing addictive behaviour, posing threats to privacy possibly resulting in fraud, corporate espionage and exposure to malware (Ferreira and Du Plessis 2009). All of the reported risks can however be minimised and managed, either on an individual or an organisational level, especially if the risks are clarified and understood in the workplace.

IBM has embraced OSN technologies and encourages employees to make use of these technologies. The investigation to determine the usage patterns and user attitudes towards the use of OSN technologies for knowledge accessing and sharing in the IBM GBS sector in South Africa revealed valuable application possibilities for organisations.

\section{Methodology design}

The research design followed an exploratory and descriptive approach using the survey method. The instruments that were used were online self-administered questionnaires and an in-depth structured interview.

A decision was reached to conduct one structured interview with a knowledge manager at IBM to gain insight into the knowledgesharing culture at IBM and how it is encouraged and supported. The interview further addressed a number of pressing questions that could not be dealt with in the questionnaire, such as how IBM identifies and manages its intellectual capital and how it manages the information and knowledge shared through the OSN tools. The interview was developed after the self-administered online questionnaires were analysed. It served to verify the responses from the questionnaires and filled some gaps after the questionnaire analyses were concluded.

The questionnaire was used to measure the opinions and perspectives of the users of OSN technologies in IBM's GBS division. Their awareness, use and preference of OSN tools, the application thereof in IBM, knowledge sharing as an organisational resource and the importance of OSN for organisations formed the constructs of the questionnaire. Consultants from offices across South Africa adhered to the criteria that had been determined for the purposive sampling. A single criterion was that a potential respondent had to use at least one of the in-house OSN tools for business purposes. A research population of 212 consultants adhered to this criterion of being active users of at least one OSN tool. According to De Vos (2002:201), for every 200 people in the research population, $32 \%$ of the population should be represented in the sample. The sample for this research was therefore aimed at 68 respondents. Fifty-six responses were received, constituting 26,4\% of the research population. However, prior to data capturing, the integrity and accuracy of the questionnaires were verified and 49 complete and valid questionnaires could be used for data analysis. The resultant $72 \%$ response rate of the initially intended sample (49 of 68 ) was consequently found to be adequate for the purpose and scope of the study.

Quantifiable responses from the closed-ended questions were analysed statistically and responses from the interview and openended questions were clustered according to identified themes. Descriptive analysis was done.

\section{Online social networking at IBM}

IBM is an international software and hardware developer and business solutions provider with one of the most advanced and envied social networking architectures in the business world to date. IBM realised the benefits of social networking early on and 
began developing their own in-house tools. This globally integrated enterprise has about 39 different social networking tools which are used throughout the company on a daily basis (Reid 2009:34). IBM's 'On Demand' workplace provides all IBM employees access to any company information they need. It provides employees with access to their own and other employees' professional profiles, making it easy to locate subject matter experts. The workplace also gives employees access to online communities of practices where employees with interests in topics can discuss these topics with their peers. The 'On Demand' environment is a place where discussion boards, instant messaging and news feeds are available, and it gives employees access to a number of services. This allows them to make 'to-do' lists, store information, bookmark important pages and generate RSS feeds on any topic, project or business news in which the employees might have an interest (Dines 2008:1). Over 398000 professionals at IBM have access to a choice of technologies they may use, based on their needs and preferences. The organisational intranet (W3 portal) provides a common user interface system that supports the company's knowledge management strategy, enabling the translation of the company's tacit knowledge into explicit knowledge and placing it in a position to identify and harness its intellectual capital. The questionnaire results shed light on the OSN awareness, usage and attitudes towards preferred tools at IBM.

\subsection{Awareness, use and preference of online social networking at IBM}

Thirty-two of IBM's in-house collaboration tools were listed and respondents were requested to indicate those that they were aware of, which of the tools they used and which of the tools the respondents preferred most (Figure 1).

Figure 1

Awareness, use and preferences of social networking tools at IBM

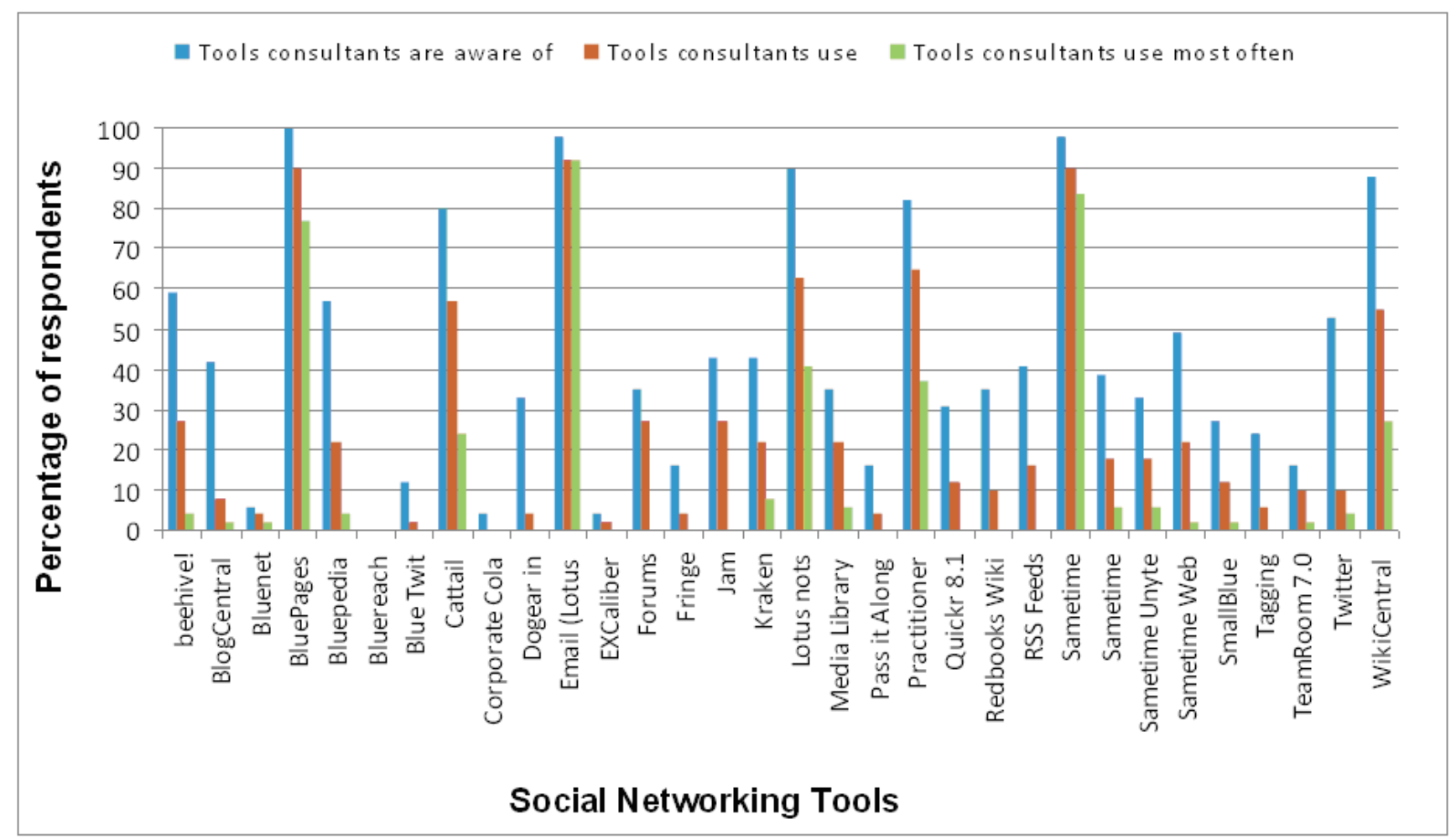

Figure 1 indicates that all the listed tools, with the exception of one tool, namely BlueReach, were familiar to respondents in varying degrees. Awareness, use and preferred use are in all cases strongly related to each other and the interviewee shed light on possible reasons why some of the tools showed poor use and preference. Tools that reflected low use and preference levels were those that were introduced in the 12 months prior to the study period as part of IBM's ongoing programme to introduce new tools and gauge their adoption rates. IBM encourages the development of new tools and tests the adoption rates internally as one methodology of assessing whether the tools could have commercial potential and whether they should be packaged for resale in the market. This means that user preferences can change over time as more people become aware of the different tools that are 
available and the benefits that each tool offers users. A tool like beehive!, IBM's internal version of Facebook, was known by almost $60 \%$ of the sample, but only $30 \%$ of the population used the tool and a very small percentage indicated it as a preferred tool. Ninety-two per cent of the respondents said that they used e-mail and selected it as one of their most preferred collaboration tools. The tool that had the second highest level of awareness and use was Sametime, IBM's Instant Messaging tool. Ninety per cent of respondents said that they used Sametime and $84 \%$ selected it as one of their most preferred tools.

\subsection{Applications of online social networking tools at IBM}

The research revealed that although OSN technologies were being investigated as an effective means of knowledge sharing, $90 \%$ of respondents indicated that they still made use of the telephone and face-to-face discussions with peers. Communities of practice were also considered to be an important part of sharing and accessing new knowledge and the results showed that respondents would rather consult people in their own networks for knowledge sharing than go to managers or consult external experts and forums. The interviewee indicated that other processes and tools used by the company are advanced and specialised and allow employees to access what they need. IBM supports different processes and practices. For example, the interviewee revealed that there were thousands of world-wide communities of practice and if none of these met the requirements of a particular subject or problem, a business justification proposal could be submitted and a new community of practice would be started. These results illustrate that although OSN tools play a major part in the sharing and accessing of knowledge, the tools have different application possibilities. It is also evident that the telephone and personal contact still play a significant role, regardless of other online possibilities.

Nevertheless, the findings illustrate the important role of OSN in the daily activities of IBM employees. Ninety-four per cent of the respondents indicated that they used OSN tools to find information, $88 \%$ of the respondents used OSN tools to communicate with others and $76 \%$ of the respondents used OSN tools to collaborate and share knowledge. Forty-seven per cent of the respondents used the OSN tools to access subject matter experts and $47 \%$ stayed informed of new developments. Six per cent of the respondents gave 'other reasons' why they used OSN tools and specified that 'communicating with colleagues on a social level' and '[l]earning and enablement - especially for global teams and cross-lines of business engagement' were important to them.

Usage patterns of OSN tools reflected positive trends for the use of such tools in the workplace. The usage frequency of OSN tools revealed that $47 \%$ of the respondents described their daily interaction as all day, every day. Forty-three per cent of the respondents used OSN tools every day, 8\% said they used the tools several times a week and none of the respondents indicated that they never used the tools.

The main themes identified for the open-ended questions in the questionnaire and the interview were clustered under the themes of Knowledge sharing as an organisational resource, Social networking and its importance for organisations and The adoption of social networking in the workplace.

\subsection{Knowledge sharing as an organisational resource}

From the questionnaire findings it is evident that OSN tools at IBM are widely used to access and share knowledge and thus create new organisational knowledge. When asked if the use of OSN tools contributed to the enhancement of knowledge sharing and learning within IBM, the interviewee said that knowledge sharing in IBM is something that is a natural part of the company culture and is powerfully encouraged by leaders within the organisation. IBM regards knowledge sharing as something that is not just natural, but as the 'heart beat' of the company. The interviewee indicated that IBM employees do not think about knowledge consciously any longer and that 'knowledge has oxygenated the project management process at IBM'. The OSN tools that are available to the company make it much easier to share and create knowledge. The OSN tools on offer allow consultants to create and build their people networks and the tools allow IBM to build their internal knowledge assets.

Knowledge assets are built through a formal process that is in place at IBM in order to identify intellectual capital through the Practitioner Portal. This is a centralised and customisable knowledge repository which consists of eight smaller specialised 
knowledge portals. Searches for specific content, subject experts and learning can be done across all eight portals or only in selected portals. IBM Global has a permanent and fairly large knowledge portal team that supports the portal. This includes processes ranging from knowledge contribution to maintenance and consistent assessments, to ensure suitability of knowledge content on the portal.

Even though intellectual capital was being traced in the organisation at the time of the study, information and knowledge sharing using OSN were not yet being managed, other than Wikis linked to certain OSNs. The interviewee did not believe that this was necessarily a flaw. Information and knowledge communication over the OSN platforms are received from employees as input that would eventually become part of a deliverable or product. The deliverable is quality assessed in terms of implementation success at clients to judge its reusability before it is regarded as a knowledge asset and included in the repository.

The essence of knowledge sharing as an organisational resource was illustrated by the interviewee using an example of a graduate who entered the company at the beginning of 2009. He had no work experience and no idea of how things worked at IBM. Within two months he had submitted his first knowledge asset (an industry-related White Paper based on his relevant postgraduate research) and identified a global technology project that he wanted to become a part of.

According to the interviewee, IBM strives to employ knowledge workers who are expected to share, to be alert, to be informed, to identify with business issues and to come up with workable solutions. As they share the knowledge they have gained by creating deliverables in the course of their work, it becomes possible for them to enter into a kind of social contract with their direct manager. Employees do not tolerate micromanagement from anyone, as they are professionals who are expert at what they do. The recognition they receive for excellence in delivery is valued in IBM.

The interviewee identified one main section where knowledge sharing could be improved: there could be greater openness between projects of a similar nature globally. The confidentially of projects makes it very difficult to determine who is working on projects or to find any information about the different projects before project closure has taken place. One has to go through many processes and people in order to find any information on live projects. Obviously confidentiality has to be adhered to but communication between the different live projects could be beneficial to greater knowledge sharing.

The interviewee referred to IBM's flexibility and principles of adopting innovation as a process, which allows workers the necessary freedom to think autonomously and come up with innovative, yet workable solutions. This approach allows users the opportunity to reach an understanding of things on their own within their networks and to be as creative and innovative as they can be. IBM says: 'You know the rules, go and play.'

The literature shows that in the knowledge-based economy 'successful enterprises are those that consistently create new knowledge, disseminate it widely throughout the enterprise and quickly embed it in new technologies and products' (Van der Walt 2003:1). According to Choo (1996:329), organisations use information in a strategic manner in three areas of the company to understand and deal with change in the environment, to create knowledge for innovation and to use information for making decisions. When people are able to understand the external environment, they can assign meaning to events and act accordingly. Knowledge creation is about the conversion of people's ideas and insights into knowledge, successfully implemented and used in turn to assist in the creation of new knowledge and products, which consequently enhances the performance of the company.

Organisations operate in a dynamic and ever-changing globally competitive environment in which they have to adapt to a variety of changes on a daily basis. For organisations to maintain their competitive positions in such a dynamic environment, it is important that they are able to gain new knowledge, distribute it wisely among users throughout the company and use the knowledge effectively to develop new technologies, processes and products to improve current operational efficiency and productivity (Van der Merwe 2004). The problem that many organisations face is gaining access to this knowledge. However, if organisations were to realise the importance of OSN technologies for knowledge sharing in their organisations, access to this knowledge would follow. 


\subsection{Social networking and its importance for organisations}

The interviewee indicated that OSN tools are used both consciously and unconsciously. Employees often use Sametime as an immediate knowledge-sharing discussion in an unconscious manner and will consciously use it to request information that they need, for instance, in locating subject matter experts or working and collaborating on projects, often on a global level. Respondents reacted to the open-ended question by indicating that they would not be able to do their jobs without the use of OSN tools. One respondent indicated that 'quite simply, without them [OSN tools] we cease to function'. The interviewee indicated that knowledge sharing is indispensible to the organisation and has become a natural process of sharing knowledge, learning and innovation while avoiding duplication.

Figure 2 indicates the extent to which respondents feel that the OSN tools enhance their knowledge-sharing patterns, the extent to which respondents feel that OSN tools promote knowledge sharing and the extent to which OSN tools contribute to the retention and protection of knowledge.

Figure 2

Value of online social networking for the business

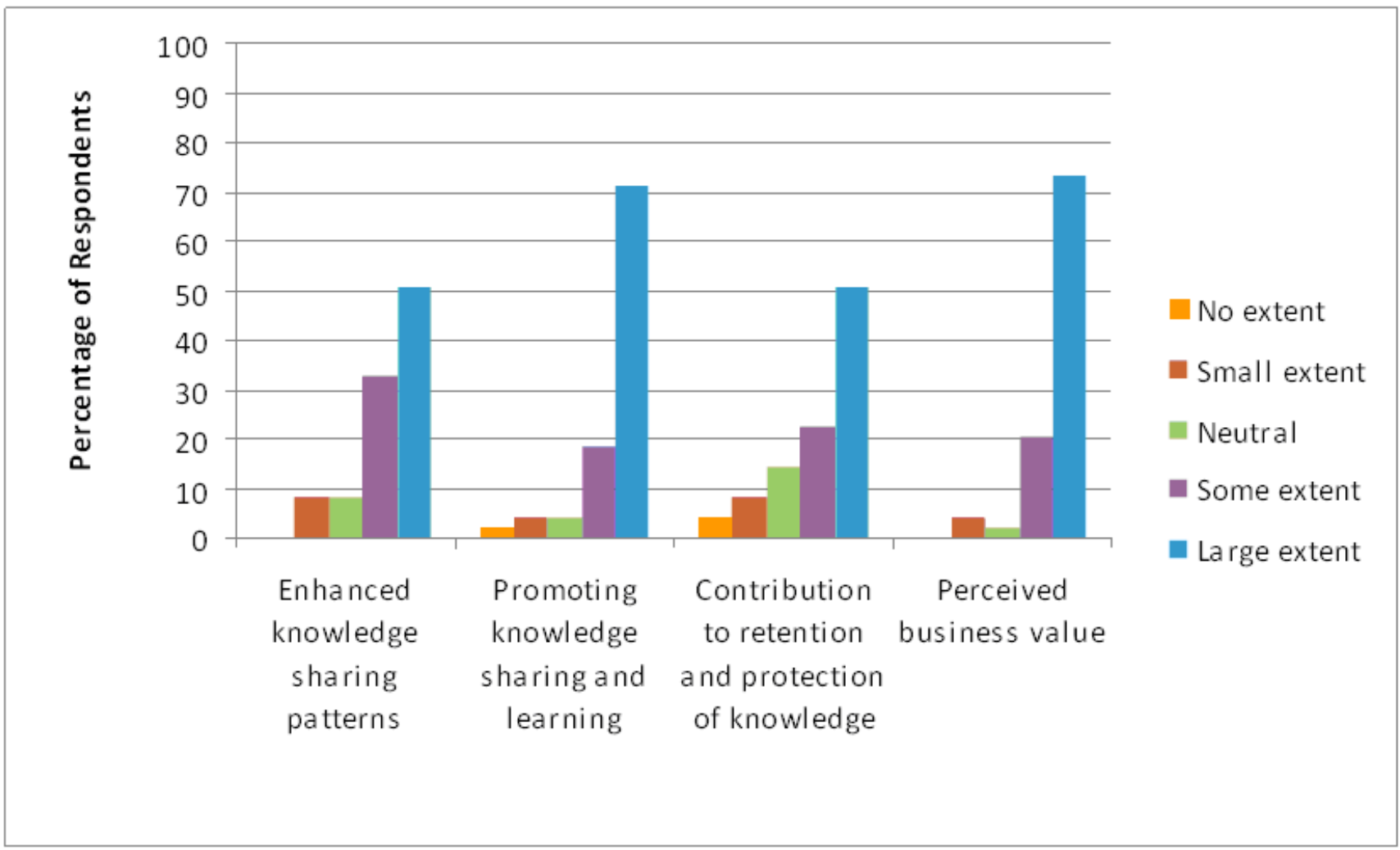

The results confirm the positive attitudes that previous questions indicated towards the use of OSN tools at IBM. Respondents found the tools an effective means of sharing and accessing knowledge: just over $50 \%$ of respondents were of the opinion that knowledge-sharing patterns are enhanced to a 'large extent' and just over 30\% felt that knowledge-sharing patterns are enhanced to 'some extent' through the use of OSN tools. More than $70 \%$ of respondents stated that the use of OSN tools promotes knowledge sharing and learning within the organisation, which not only shows that consultants at IBM feel that they are able to share knowledge through the use of OSN tools but also that respondents have a positive attitude towards the use of OSN tools. Respondents were of the opinion that OSN is useful in a business sense. A strong link exists between how beneficial OSN tools are in their line of work and whether they perceive OSN to have business value. Seventy-three per cent of the respondents indicated that OSN had business value to a 'large extent'. 
IBM is a globally integrated enterprise (GIE) and respondents were requested to indicate to what extent they thought IBM could operate as a GIE without the use of OSN tools. The combined results for 'no extent' and 'small extent' revealed that $78 \%$ of respondents held the opinion that IBM could not operate effectively as a GIE without the use of OSN tools, proving that IBM has developed a culture where knowledge sharing, and especially knowledge sharing using OSN tools, is promoted and the use of these tools has become a natural part of business for IBM consultants. IBM might be interested in investigating why $16 \%$ of the respondents believed that IBM could operate effectively as a GIE without OSN tools.

IBM therefore realises that its most important company asset is its employees. The company has developed a platform for them to express themselves and develop their knowledge, which in turn results in greater intellectual capital for the entire organisation. The literature points out that value creation in the dynamic knowledge economy is becoming increasingly reliant on the 'manipulation of information, knowledge, ideas, expertise and relationships which are collectively known as intellectual capital' (Van Zyl 2006:70). According to Liebowitz (2007:11), social networks serve as 'incubators' where new ideas can grow and later be turned into goods and services that will be sold for profit. Organisations that make use of social networks will quickly realise the benefits of employees sharing their ideas and collaborating with others through the use of these tools, as social networks play a vital role in the alignment of a number of disparate ideas within the organisation, improving the process of knowledge sharing and generation of new ideas (Liebowitz 2007:11).

\subsection{Adoption of social networking in the workplace}

According to the interviewee 'there is no perception in IBM that the OSN tools are abused. Consultants in GBS have a very good work ethic and commitment to their jobs.' The interviewee further stated that because of IBM's open and honest communication policy throughout the company, there is very little that can be done 'on the sly' and that the mindset of employees when using the social networking tools is business-focused. OSN tools are reported to be useful in helping IBM employees deliver products and complete projects on time. Employees receive incentives in the form of points for their knowledge contributions and knowledge activities such as ratings from peer reviews, comments, downloads or views from the knowledge portal. Managers are informed of these points for knowledge activities which are considered in the annual performance assessment. Rewards such as better bonuses, free merchandise (Thank you! Programme), salary increments, promotions and global recognition in IBM incentivise employees to share knowledge. The interviewee indicated that consultants brand their expertise and market it in IBM, wanting to be the 'go to' person for a particular process or business sector. They want to share their knowledge and in many instances cannot do their work without sharing knowledge.

Many companies have not incorporated OSN tools into their organisations out of fear that the organisation's content and proprietary information will be shared with external parties, which could lead to the downfall of the organisation (McClure 2009:22). OSN tools are often based outside the company firewall, on browser-based applications on the Web. The threat of company information being leaked using these tools is too real for many employers and was stated as one of the reasons for hesitating to introduce OSN tools into the workplace (McClure 2009:22). In the case of IBM, all OSN tools are used in-house and located on a secure practitioner portal. In addition, administrative processes such as reminders to protect proprietary information and the signing of contracts annually to comply with IBM guidelines are used to manage the awareness of employees to avoid inadvertent disclosure of information at all times.

\section{Conclusion}

Although the use of online social network (OSN) tools in the workplace is still not widely accepted, it is becoming an important and possibly unavoidable part of the business world. The study conducted at IBM has shown that the use of OSN tools is indeed an effective way of sharing knowledge and learning within the enterprise that can serve as an example for other organisations.

Employees in IBM's GBS division described how they cannot function without the use of social networking tools. Many of the respondents explained how they rely on OSN tools every day in order to do their jobs. Since the employees' contributions are effectively documented through the use of these tools, the organisation's intellectual capital can be harnessed as an organisational 
resource. IBM has consequently realised how important OSN is and how it enhances the effectiveness of knowledge sharing in the organisation. Almost seamless adoption of the technologies was possible because employees swiftly witnessed the benefits of OSN. Through a reward system that measures knowledge activities, both hesitant and enthusiastic employees received incentives to contribute.

The concern that many employers have about their company's private information being leaked or their employees wasting time by using the tools for social purposes reminds of the misconception when telephones were first introduced to every office desk and it was feared that they would be abused for personal purposes. The telephone has become an indispensible office tool. In a similar vein, respondents corroborated the acceptance of OSN tools to the point where they stated that they could not conceive themselves doing their work effectively without it.

With the correct management and awareness creation, individuals and organisations will sufficiently sensitise users to the effective use of OSN in a corporate setting. The study conducted at IBM has shown that OSN technologies can be applied effectively in an organisation. IBM, being an organisation from the information and communications technology industry, is probably likely to show greater acceptance of new technologies and it is not known how individuals in other industries will grapple and cope with new technologies. Further case study research is recommended in a variety of industries in order to identify and compare different types of organisations that have implemented the use of OSN technologies. The benefits and problems experienced by each can be identified to find applicable solutions where necessary. Keeping up with the tempo of change and managing resources amid the change could present itself as the real reason for resistance to embrace new technologies.

OSN has proved to be an effective platform where the individuals in the IBM enterprise know what it knows, use what it knows to its advantage and quickly know something new in order to be innovative and gain competitive advantage in a global economy driven by knowledge.

\section{Acknowledgement}

Many thanks to Sharon van Biljon who balanced the academic views with a practitioner's input.

\section{References}

Amidon, D.M., 1999, 'Knowledge innovation', viewed 17 October 2009, from http://www.entovation.com/innovation/knowinno.htm.

Brooking, A., 1996, Intellectual capital, Berkshire House, London.

Business Dictionary, 2009, 'Innovation', $\quad$ viewed $17 \quad$ October 2009, from http://www.businessdictionary.com/definition/innovation.html.

Choo, C.W., 1996, 'The knowing organization: how organizations use information to construct meaning, create knowledge and make decision', International Journal of Information Management 16(5), 329-340.

Chua, A., 2003, 'Knowledge sharing: a game people play', Aslib Proceedings 55(3),117-129.

Dines, D., 2008, 'IBM's strategy for taking social networking to the enterprise: an inside look at lotus connections', viewed 12 May 2009, from http://download.boulder.ibm.com/ibmdl/pub/software/cn/web20/Wainhouse IBM Strategy.pdf.

Clarke, T., 2001, 'The knowledge economy', Education and Training 43(4/5), 189-196.

De Vos, A.S. (ed.), 2002, Research at grass roots: for the social sciences and human service professions, 2nd edn., Van Schaik, Pretoria. 
Ferreira, A. \& Du Plessis, T., 2009, 'Effect of online social networking on employee productivity', South African Journal of Information Management 11(1).

Goetchius, A., 2008, Career building through social networking, The Rosen Publishing Group, New York.

Liebowitz, J., 2007, Social networking: the essence of innovation, Scarecrow Press Inc., Lanham Maryland.

McClure, M., 2009, 'Creating safe, collaborative cultures in a Web 2.0 world', EContent Magazine, 1 June, p. 22.

Prusak, L., 1996, 'The knowledge advantage', Strategy and Leadership 28(1), 6-8.

Reid, C.K., 2009, 'Should business embrace social networking?', EContent Magazine, 15 June, p. 34.

Schneckenberg, D., 2009, 'Web 2.0 and the shift in corporate governance from control to democracy', Knowledge Management Research and Practice 7(3), 234-248.

Van der Merwe, A., 2004, 'Managing intranets to improve knowledge sharing', South African Journal of Information Management $6(4), 1-13$.

Van der Walt, C., 2003, 'Knowledge sharing via enterprise intranets', unpublished Masters dissertation, University of Johannesburg.

Van Zyl, C.R., 2006, 'Intellectual capital and marketing strategy intersect for increased sustainable competitive advantage', unpublished Master's dissertation, University of Johannesburg.

Warr, W.A., 2008, 'Social software: fun and games, or business tools?', Journal of Information Science 34(4), 591-604.

Zhou, A. \& Fink, D., 2003, 'The intellectual capital Web', Journal of intellectual capital 4(1), 34-48. 